\title{
A CRITICAL FORCE CONSTANT OF THE STRUCTURAL PHASE TRANSITION IN QUARTZ
}

\author{
J.A. MARTIN-PEREDA*, C.Y. SHE \\ Department of Physics, Colorado State University, Fort Collins, Colorado 80521, USA \\ and \\ D.F. EDWARDS \\ Departments of Electrical Engineering and Physics, Colorado State University, Fort Collins, Colorado 80521, USA
}

Received 3 October 1972

\begin{abstract}
Long wavelength optical phonons of quartz were analyzed by a Born-Von Karman model not previously used. It was found that only one force constant associated with the turning of the $\mathrm{Si}-\mathrm{O}$ bonds has a critical effect on the softmode frequency and the $\alpha-\beta$ transition in quartz. The square of the soft-mode frequency was found to depend linearly on this force constant which has the temperature dependence $k(T)=-5.33+225.3 \times 10^{-4}(851-T)^{2 / 3}$ in units of $10^{4} \mathrm{dyn} / \mathrm{cm}^{2}$.
\end{abstract}

The $\alpha \mu \beta$ structural phase transition of crystalline quartz at $T_{\mathrm{C}}=573^{\circ} \mathrm{C}$ has long been investigated [1] and the lowest symmetric optical phonon mode at $207 \mathrm{~cm}^{-1}$ has been thought to be relevant in a major way [2]. This relation was confirmed when Kleinman and Spitzer [3] showed that the eigenvector of this lowest (soft) mode is very similar to the atomic displacements during the $\alpha-\beta$ transition in quartz. Due to the complication that a weak anomalous mode existed nearby, the temperature dependence of the phonon frequency was measured only recently [4]. Scott [5] has attributed this anomaly to a two-phonon mode which couples with the soft mode in question. When decoupled, the temperature dependence of the softmode frequency was determined as $\omega=26\left(T_{0}-T\right)^{1 / 3}$ $\mathrm{cm}^{-1}$. The quartz transition is "slightly first order" which exhibits hysteresis effects [4]; the stability limit $T_{0}$ may differ from the transition temperature $T_{\mathrm{c}}$.

Owing to the complex structure of quartz with nine atoms per primitive cell, the progress in theoretical calculation has been lacking even though they were started early [6]. More recently, Elcombe [7] has made an analysis from the viewpoint of lattice dynamics considering both Born--Von Karman and rigid ion models. The harmonic approximations were used in

\footnotetext{
* Present address: E.T.S. Ingenieros de Telecomunicacion, Ciudad Universitaria, Madrid-3, Spain.
}

all these works $[3,7]$ and the force constants (parameters) involved in the models were determined by fitting the calculated phonon frequencies to measured values at room temperature. So far, no serious attempt has been made to follow the development of the soft mode as the transition temperature is approached.

We report here calculations carried out with a different Born-Von Karman model using five adjustable force constants. Like previous works, these parameters were determined by fitting the room temperature phonon frequencies. In this respect, our model is equivalent to Elcombe's. However, by examining the softmode motion, we were able to single out the force constant, $\kappa$, associated with the turning of the Si-O bonds as the critical parameter (in our model) responsible for the $\alpha-\beta$ structural phase transition in quartz. Thus, in this paper, we report the calculation of phonon frequencies as a function of the critical force constant, $\kappa$, and show that only the soft-mode frequency is affected significantly by this force constant. It is found that the square of the soft-mode frequency, $\omega^{2}$, depends linearly on $k$. Using the experimental softmode frequencies, a simple power law was determined for $k$ as a function of $\left(T_{0}-T\right)$. This microscopic critical parameter $k$ may be related to the phenomenological displacement parameter $\Delta x_{0}$ proposed recently by Höchli and Scott [8] who have determined the stability limit to be $T_{0}=578^{\circ} \mathrm{C}$. 
Our normal mode calculation with harmonic approximations is standard [9]. Only the short-range interactions (Born-Von Karman model) of Si-O and $\mathrm{O}-\mathrm{O}$ bonds are considered. Due to the symmetry of $\mathrm{SiO}_{4}$ tetrahedron, the force constant matrices of these bonds may be expressed as:

Si-O: $\left(\begin{array}{ccc}k & \kappa & k \\ \kappa & k & k \\ \kappa & \kappa & k\end{array}\right)$, and O-O: $\left(\begin{array}{ccc}a & c & 0 \\ c & a & 0 \\ 0 & 0 & b\end{array}\right)$.

The principal vibrations of the soft mode involves oxygen atoms [3]; the direction of these motions are depicted in fig. 1 . It is clear that the dominant motion is the turning of the Si-O bonds and suggests that the force constant $k$ is responsible for the temperature dependence of the soft-mode frequency. Other force constants have only minor effects.

In our calculation, we follow the procedures as outlined by Lacina [9]. Symmetry coordinates [10] were first obtained and then the dynamical matrices in terms of the above mentioned five force constants are grouped according to the symmetry species of the point group of quartz [11]. Like Elcombe [7], the
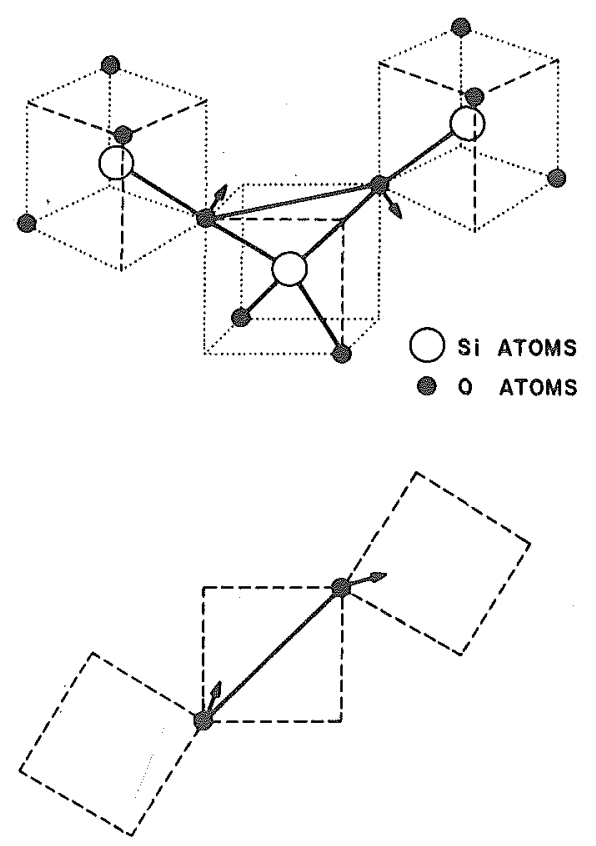

Fig. 1. Principal motion of the soft mode and its projection on to the basal plane. perfect tetrahedron was used in the model and thus no distinction between $\alpha$ and $\beta$ quartz was made as far as the model is concerned. The values of the adjustable force constants would, of course, be different in the two phases. Eigenvalues and eigenvectors for long wavelength optical phonons were calculated using different values for the five force constants in the model. The following set of force constants, $k=-5.7, \kappa=$ 4.0, $a=1.2, b=15.7$, and $c=15.0$, all in units of $10^{4} \mathrm{dyn} / \mathrm{cm}^{2}$, are found to give a good fit to all experimental optical phonon frequencies $\left(\mathrm{A}_{1}\right.$ and $\mathrm{A}_{2}$ modes, as well as transverse $E$ modes) at the room temperature. The quality of the fit is comparable to that obtained by Kleinman and Spitzer [3] or Elcombe [7].

Keeping the values of $k, a, b$, and $c$ constant, we found that the soft-mode frequency can vary from $6.1 \times 10^{12} \mathrm{~Hz}$ to zero by varying the force constant $\kappa$ alone. Shown in fig. 2 are the optical phonon frequencies of the $A_{1}$ and $A_{2}$ modes as a function of $k$. Notice the frequency of all other modes except the soft mode remains relatively constant. This means that the force constant $\kappa$ indeed is responsible for the $\alpha-\beta$ structural phase transition in quartz. Also, we have found little effects of $k$ on the $E$ modes (not shown). Furthermore, variation on other force constants have been numerically investigated. They all gave undesirable effects as expected. For example, changing $k$ while keeping other parameters constant resulted in significant changes in phonon frequencies of nearly all modes. The best fit to the soft-mode frequency shown in fig. 2 is $\omega=172.6(5.33+\kappa)^{0.49} \mathrm{~cm}^{-1}$, where $\kappa$ is in units of $10^{4} \mathrm{dyn} / \mathrm{cm}^{2}$. Approximately, we find that $\omega^{2}$ depends on $\kappa$ linearly as

$\omega^{2}=3 \times 10^{4}(5.33+\kappa) \mathrm{cm}^{-2}$.

Using the experimental data of Scott [5] and of Shapiro and Cummins [4], we can express the temperature dependence of the critical force constant in units of $10^{4} \mathrm{dyn} / \mathrm{cm}^{2}$ as

$\kappa(T)=-5.33+225.3 \times 10^{-4}(851-T)^{2 / 3}$

To associate a temperature dependence to a harmonic force constant, such as $\kappa$, amounts to solving the problem with a pseudo-harmonic approximation. The origin of the temperature dependence comes, of course, from the anharmonic interactions in the crystal. To theoretically justify the temperature depend- 

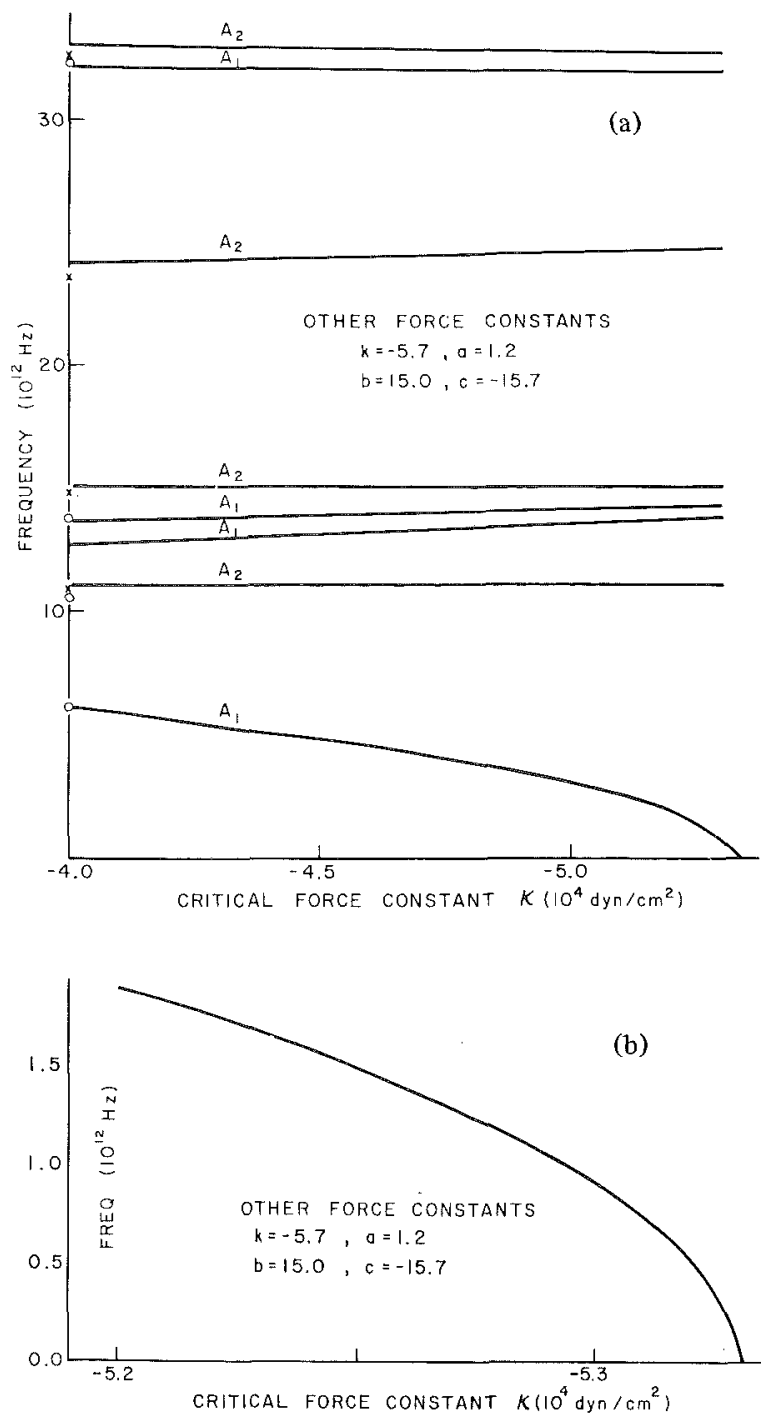

Fig. 2. Optical phonon frequencies as a function of the critical force constant $k$. (a) All $A_{1}$ and $A_{2}$ modes, and (b) the soft mode plotted on expanded scale near the transition region. 0 and $X$ are the experimental frequencies (at room temperature) of the $A_{1}$ and $A_{2}$ modes, respectively.

ence in eq. (2), a treatment with either a random phase approximation or a self-consistent phonon approximation [12] should be made. For a complicated crystal-like quartz, such a task would be very much involved and is beyond the scope of this paper. Our aim here is to point out the importance of the critical force constant $\kappa$ in relation to the soft-mode frequency and the $\alpha-\beta$ transition in quartz. The linear dependence of $k$ on $\omega^{2}$ in eq. (1) is anticipated; it is physically sound. The experimental temperature dependence of the soft-mode frequency may be accounted for when the relation, eq. (2), is used. Höchli and Scott [8] discussed their experimental results in terms of a displacement parameter associated with the rotation of $\mathrm{SiO}_{4}$ tetrahedra by following the phenomenological development of Landau and Lifshitz [13]. They then obtained the relationship between the soft-mode frequency and the displacement parameter as $\omega \propto \Delta x_{0}$. The turning of the Si-O bonds and the two-fold rotation of $\mathrm{SiO}_{4}$ tetrahedra are physically related. By comparing the form of the energy expression in the two cases, the temperature-dependent change of our critical force constant may be related to their displacement parameter as $\kappa(T)-\kappa\left(T_{0}\right) \propto \Delta x_{0}^{2} \propto\left(T_{0}-T\right)^{2 / 3}$.

The authors wish to acknowledge the helpful discussions with Professor J.C. Raich. One of us (J.A. M-P) wishes to thank Professor Segovia for granting him a short leave from ETSIT to complete this work.

\section{References}

[1] I.A. Yakovlev, L.F. Mikheeva and T.S. Velichkina, Kristallografiya 1 (1956) 123 [Soviet Phys., Crystallography 1 (1956) 91)].

[2] B.D. Saksena, Proc. Indian Acad. Sci A12 (1940) 93; T.M.K. Nedungadi, ibid. A11 (1940) 86.

[3] D.A. Kleinman and W.G. Spitzer, Phys. Rev. 125 (1962) 16.

[4] S.M. Shapiro, D.C. O'Shea and H.Z. Cummins, Phys. Rev. Lett. 19 (1967) 361; S.M. Shapiro and H.Z. Cummins, in: Light scattering spectra of solids, ed. G.B. Wright (Springer-Verlag, New York 1969) p. 705.

[5] J.F. Scott, Phys. Rev. Lett. 21 (1968) 907.

[6] B.D. Saksena, Proc. Indian Acad. Sci. A16 (1942) 270; ibid. A22 (1945) 379.

[7] M.M. Elcombe, Proc. Phys. Soc. 91 (1967) 947.

[8] U.T. Höchli and J.F. Scott, Phys. Rev. Lett. 26 (1971) 1627.

[9] W.B. Lacina, Harvard University, Technical Report No. ARPA-36, March 1969.

[10] E.B. Wilson, J.C. Decius and P.C. Cross, Molecular vibrations (McGraw-Hill Book Co., New York 1955).

[11] J.A. Martin-Pereda, Doctoral dissertation, 1971, E.T.S. Ingenieros de Telecomunicacion, Madrid, Spain (unpublished).

[12] N.R. Weithamer, Phys. Rev. B1 (1970) 572.

[13] L.D. Landau and E.M. Lifshitz, Statistical Physics, 2nd ed. (Addison-Wesley Publ.Co., Inc., Reading, Mass., 1969) Ch. 14. 